Manel BoudouAYA ${ }^{1}$

Hachemi BenHASSAINI ${ }^{2}$

\title{
Évaluation de la variabilité de la couleur du bois de Pistacia atlantica Desf.
}

Fatima Zohra BENDIMERED-MOURI ${ }^{2}$

Frédéric MOTHE ${ }^{3}$

Meriem FOURNIER ${ }^{4}$

${ }^{1}$ Université Djilali Liabès de Sidi Bel Abbès

Faculté des Sciences de la Nature

et de la Vie

Laboratoire d'écodéveloppement

des espaces

Cité rectorat, route de Tlemcen

BP 89, Sidi Bel Abbès (22000)

Algérie

${ }^{2}$ Université Djilali Liabès de Sidi Bel Abbès

Faculté des Sciences de la Nature

et de la Vie

Laboratoire de biodiversité végétale :

conservation et valorisation

Cité rectorat, route de Tlemcen

BP 89, Sidi Bel Abbès (22000)

Algérie

${ }^{3}$ Inra

UMR 1092 LERFoB

Rue Amance

54280 Champenoux

France

${ }^{4}$ AgroParisTech

UMR 1092 LERFoB

54000 Nancy

France

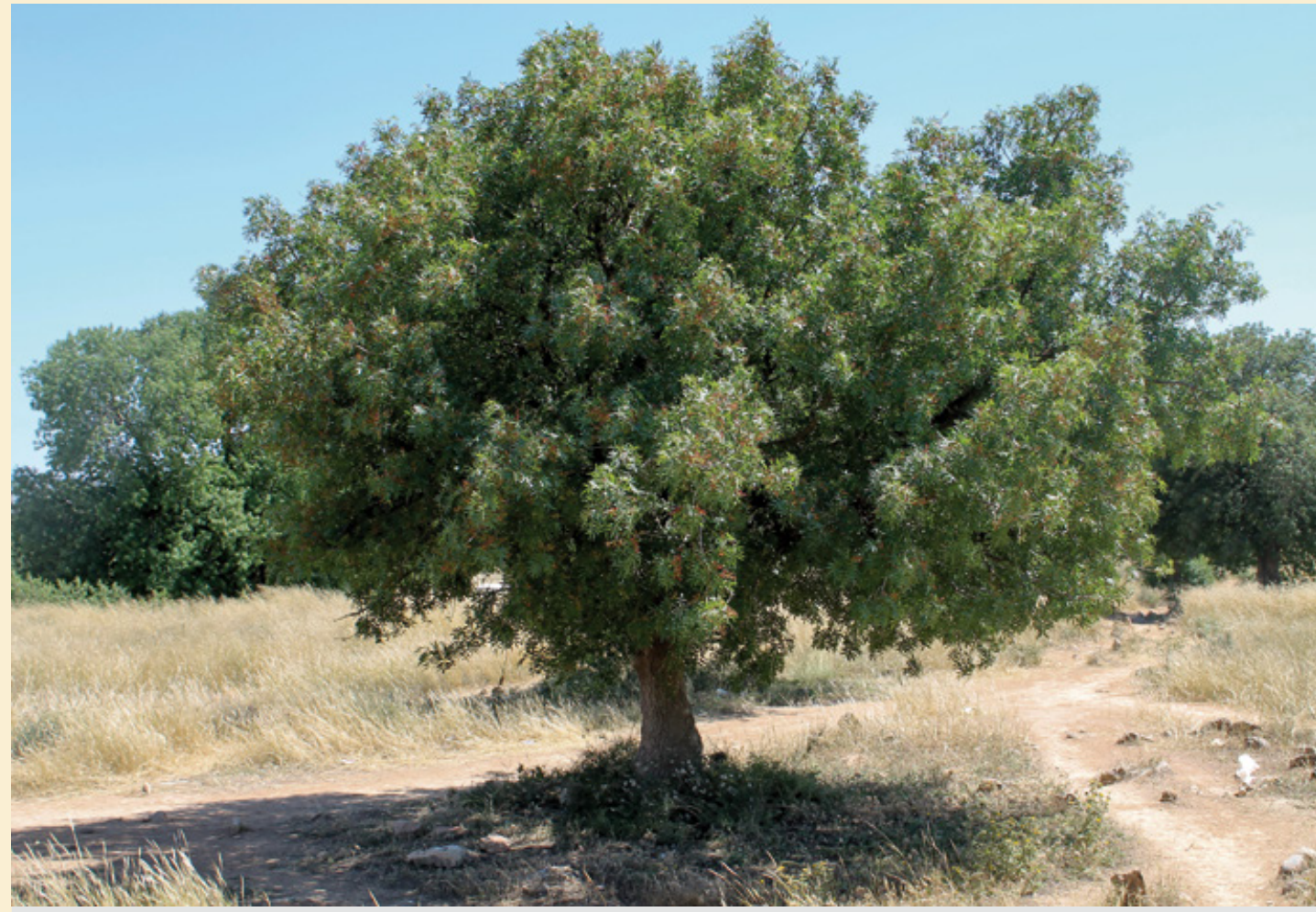

Photo 1.

Aspect général d'un pistachier de l'Atlas aux environs de Sidi Bel Abbès (Nord-Ouest de l'Algérie).

Photo M. Boudouaya. 


\section{RÉSUMÉ}

\section{ÉVALUATION DE LA VARIABILITÉ DE LA COULEUR DU BOIS DE PISTACIA ATLANTICA DESF. DU NORD DE L'ALGÉRIE}

Parmi les propriétés caractérisant le bois des essences naturelles et particulièrement celles des zones arides et semi-arides, la variabilité de la couleur conditionne leur utilisation en tant que matériau apparent de haute valeur ajoutée, en menuiserie-ébénisterie, par exemple. Les travaux présentés ici ont pour but de contribuer à la valorisation esthétique du bois d'une espèce ligneuse répandue en Algérie mais très peu étudiée, le pistachier de l'Atlas, Pistacia atlantica Desf. La variabilité de la couleur a été évaluée sur des éprouvettes de bois coupées dans l'aubier et le duramen d'arbres provenant de deux régions différentes du point de vue bioclimatique en Algérie nord-occidentale. L'évaluation de la couleur du bois a été effectuée au sein du Laboratoire d'étude des ressources forêt-bois (LERFoB) à Nancy, France, en utilisant un spectrocolorimètre et un logiciel pour la mesure et l'affichage des données. Les résultats obtenus indiquent la présence de variation de la couleur du bois du pistachier de l'Atlas entre les deux régions (pour les trois composantes chromatiques, $L^{*}, a^{*}$ et $b^{*}$ ) et entre les arbres (pour la composante $L^{\star}$ ). L'aubier, avec son bois plus clair, présente des composantes colorimétriques, particulièrement la clarté $\left(L^{*}\right)$ et la composante vert-rouge $\left(a^{\star}\right)$, très différentes de celles du duramen. Nous en déduisons que les facteurs du milieu ont une influence sur les caractéristiques colorimétriques du bois de cette espèce, en plus des caractéristiques individuelles intrinsèques de chaque arbre. Enfin, la clarté $L^{*}$ et les composantes vert-rouge $a^{*}$ et bleu-jaune $b^{*}$ présentent des variations entre les différents types de bois de pistachier.

Mots-clés : Pistacia atlantica Desf., zone de transition, CIELab, espace colorimétrique, duramen, aubier, Algérie nord-occidentale.

\section{ABSTRACT}

\section{ASSESSMENT OF COLOUR VARIATIONS IN PISTACIA ATLANTICA DESF. WOOD FROM NORTHERN ALGERIA}

Among the characteristic properties of timber from natural tree stands, particularly those in arid and semi-arid zones, variations in colour are decisive in the uses made of the wood as a high added-value visible material, in joinery and cabinet-making for example. This study was conducted to help promote the aesthetic value of a woody species which is common in Algeria but under-researched, the Mt. Atlas mastic tree, Pistacia atlantica Desf. Variations in wood colour were assessed from samples cut from the sapwood and heartwood of mastic trees from two different bioclimatic regions in north-western Algeria. The colour assessments were made at the Forest and Timber Resources Laboratory (LERFoB) in Nancy, France, using a spectrophotometer with measurement and display software. The results indicate variations between the two regions in the colour of Mt. Atlas mastic tree wood (for the three chromatic components $L^{*}$, $a^{*}$ and $b^{*}$ ) and among the different trees (component $\left.L^{*}\right)$. The colorimetric components of the lighter-coloured sapwood, especially brightness $\left(L^{*}\right)$ and the red-green component $\left(a^{\star}\right)$, are very different to those of the heartwood. From this we infer that environmental factors have an influence on the colorimetric characteristics of the wood in this species, as well as the intrinsic individual characteristics of each tree. Finally, brightness $\left(L^{*}\right)$ and the red-green $\left(a^{\star}\right)$ and blue-yellow $\left(b^{\star}\right)$ components vary between the different types of mastic tree wood.

Keywords: Pistacia atlantica Desf., transition zone, CIELab, colour space, heartwood, sapwood, north-western Algeria.

\section{RESUMEN}

\section{EVALUACIÓN DE LA VARIABILIDAD DEL COLOR EN MADERA DE PISTACIA ATLANTICA DESF. DEL NORTE DE ARGELIA}

Entre las propiedades que caracterizan la madera de especies naturales, especialmente las de zonas áridas y semiáridas, la variabilidad de color condiciona su uso en elementos de madera vista de gran valor añadido, como en carpintería y ebanistería, por ejemplo. El objetivo de este estudio es contribuir al valor estético de una especie leñosa, común en Argelia pero muy poco estudiada, el almácigo del Atlas, Pistacia atlantica Desf. Se evaluó la variabilidad del color mediante probetas de albura y duramen de árboles provenientes de dos regiones bioclimáticas distintas de Argelia noroccidental. La evaluación del color de la madera se llevó a cabo en el Laboratoire d'Étude des Ressources Forêt-Bois (LERFoB) en Nancy, Francia, empleando un espectrocolorímetro y un software para la medida y visualización de datos. Los resultados obtenidos indican variaciones de color en la madera de almácigo entre las dos regiones (entre los tres componentes cromáticos $L^{*}, a^{\star}$ y $b^{\star}$ ) y entre los árboles (en el componente $L^{\star}$ ). La albura, con su madera más clara, presenta componentes colorimétricos, especialmente la claridad $\left(L^{*}\right)$ y el componente verde-rojo $\left(a^{\star}\right)$, muy diferentes a los del duramen. De ello se deduce que, además de las características individuales propias de cada árbol, los factores del medio influyen en las características colorimétricas de la madera de esta especie. Por último, la claridad $L^{*}$ y los componentes verde-rojo $a^{*}$ y azul-amarillo $b^{*}$ presen$\tan$ variaciones entre los distintos tipos de madera de almácigo.

Palabras clave: Pistacia atlantica Desf., área de transición, CIELab, espacio colorimétrico, duramen, albura, Argelia noroccidental. 


\section{Introduction}

Le pistachier de l'Atlas Pistacia atlantica Desf. est un taxon vivace et rustique pouvant vivre plusieurs siècles et bien acclimaté aux conditions édaphiques et climatiques extrêmes. C'est un arbre xérophile, dioïque, à tronc bien individualisé et cime hémisphérique. Cette essence fait partie des ressources méconnues dans le monde. En Algérie, l'espèce est dévalorisée et délaissée, et rares sont les études consacrées à ce taxon. Son existence dans certains endroits, sous forme de reliques isolées ou groupées, reflète sa position d'arbre de climax naturel et non pas de para-climax monospécifique (Monjauze, 1980) (photo 1). Cet écodème, d'après la terminologie de Gillet (2000), nous informe sur la préexistence d'anciennes forêts claires ou de steppes arborées (Quézel et al., 1999 ; Quézel, 2000). Les trois plus gros arbres de cette espèce ont été repérés depuis environ trois décennies par Monjauze (1980) dans la région de Sidi Bel Abbès, au Nord-Ouest de l'Algérie, qui est précisément la zone où a été effectué notre échantillonnage. Nombre de coproduits et d'avantages sont tirés du pistachier de l'Atlas. Son bois est depuis longtemps utilisé comme bois d'œuvre artisanal et est considéré malheureusement comme un excellent bois de carbonisation (Monjauze, 1980).

La couleur est un caractère esthétique important pour l'emploi du bois en menuiserie et surtout en ébénisterie. Le bois de chaque essence a une couleur qui lui est propre, souvent plus sombre dans le bois de cœur, et se modifie lentement à l'air et à la lumière (Zanetti et al., 2003). La couleur peut également varier suivant les arbres et les provenances. Le bois de chêne, par exemple, est d'une teinte variant du brun clair au gris brun ou au brun rougeâtre (Dumon, 1980). La couleur du bois dépend également du mode de débit, de sa direction par rapport aux trois axes du plan ligneux, de la composition chimique, de la nature des sources lumineuses et de l'orientation de la surface par rapport à ces sources lumineuses (Janin, 1987). La colorimétrie est la mesure objective et quantitative de la couleur d'un objet en vue de son repérage dans un système de représentation conventionnel des couleurs. Elle qualifie scientifiquement et rigoureusement la perception de la couleur et permet de traduire la couleur en valeurs numériques (Chrisment, 1997). L'étude de la variabilité de la couleur du bois est récente. Cet aspect esthétique est très important pour évaluer la qualité d'un produit surtout dans l'ameublement, la parqueterie ou le tranchage (Mazet et Janin, 1990). Kokutse (2002) a signalé que la mesure physique de la couleur, rarement pratiquée sur les espèces tropicales, est souvent utilisée chez les espèces tempérées comme outil de tri de la qualité ou comme indicateur d'autres propriétés comme la durabilité naturelle.

Cette étude a pour but de contribuer à la valorisation esthétique du pistachier de l'Atlas, en mettant l'accent sur la couleur, alors que les autres aspects physiques et mécaniques nécessaires à cette valorisation sont en cours d'étude. Ce travail est le premier du genre à aborder cette problématique sur cette essence.

\section{Matériel et méthodes}

\author{
Matériel végétal
}

\section{Travail de terrain}

Les échantillons de bois ayant fait l'objet d'une évaluation colorimétrique ont été prélevés sous forme d'éprouvettes à partir de rondelles coupées sur six arbres provenant de deux régions différentes, à raison de trois arbres par région (photo 2). Sur chaque arbre, des mesures dendrométriques ont été effectuées au mètre ruban dont les principales caractéristiques sont exposées dans le tableau I.

Les deux régions sont différentes du point de vue bioclimatique en Algérie nord-occidentale (figure 1). La première provenance est la région de Sidi Bel Abbès, caractérisée par un bioclimat méditerranéen semi-aride à influence continentale et à deux saisons bien distinctes : l'hiver humide et froid, l'été sec et chaud (Direction des services agricoles de Sidi Bel Abbès, 2008). La seconde provenance est la région de Tiaret, à bioclimat semi-aride transitionnel entre le climat aride du Sahara et le climat méditerranéen de la côte avec des pluies concentrées pour l'essentiel sur la période hivernale (Abdelli, 2002). Les séries de données pluviométriques relatives à la période 1952 jusqu'à 2011, enregistrées aux stations météorologiques des deux régions, révèlent que la moyenne des précipitations annuelles est égale à $453 \mathrm{~mm}$ pour la région de Tiaret et $350 \mathrm{~mm}$ pour Sidi Bel Abbès (Agence nationale des ressources hydrauliques, 2013).

\section{Découpe des billons}

Dans chacun des six arbres échantillonnés, un billon de $50 \mathrm{~cm}$ de longueur à $1,30 \mathrm{~m}$ de hauteur du sol a été débité à l'endroit du sondage des carottes de sélection ; une rondelle de $15 \mathrm{~cm}$ d'épaisseur a été prélevée à la base de chaque grume, comme l'illustre la figure 2 .

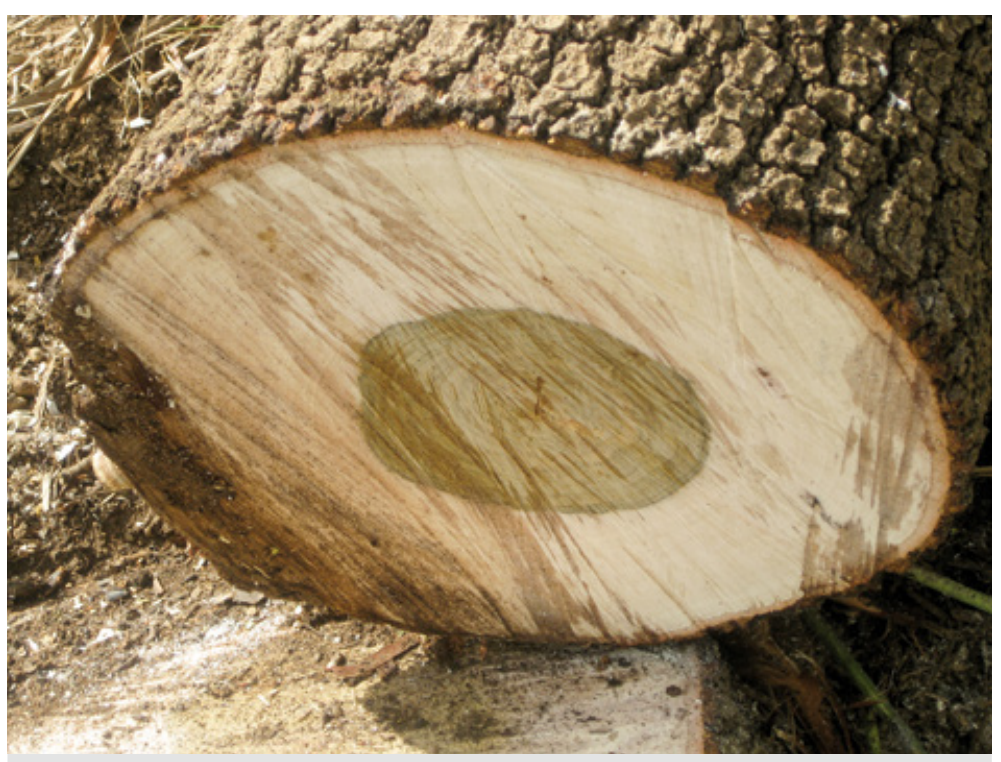

Photo 2.

Billon coupé d'un pistachier de l'Atlas.

Photo M. Boudouaya. 


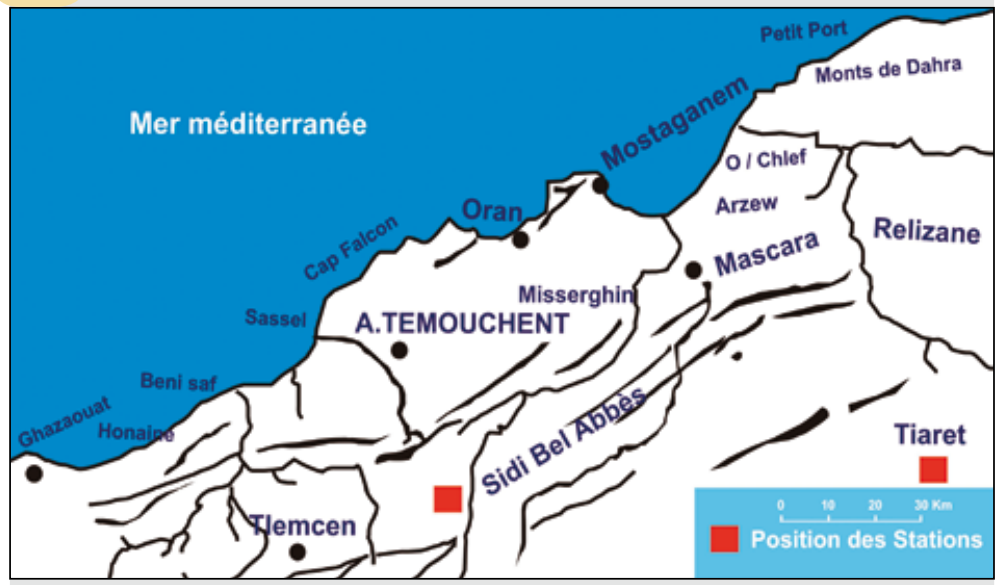

Figure 1.

Localisation des deux régions de prélèvement des échantillons (Nord-Ouest de l'Algérie).

Chacun des billons a été marqué d'une lettre indiquant sa provenance, $T$ (pour Tiaret) et $S$ (pour la région de Sidi Bel Abbès), suivie par un numéro de 1 à 3 indiquant le numéro de l'arbre. Le premier arbre choisi a été désigné par le numéro 1. Par exemple, S2 désigne le deuxième arbre prélevé de la région de Sidi Bel Abbès.

\section{Travail de laboratoire}

Tous les essais ayant fait l'objet de ce travail ont été réalisés au Laboratoire d'étude des ressources forêt-bois (LERFoB) à l'Inra de Nancy (France).

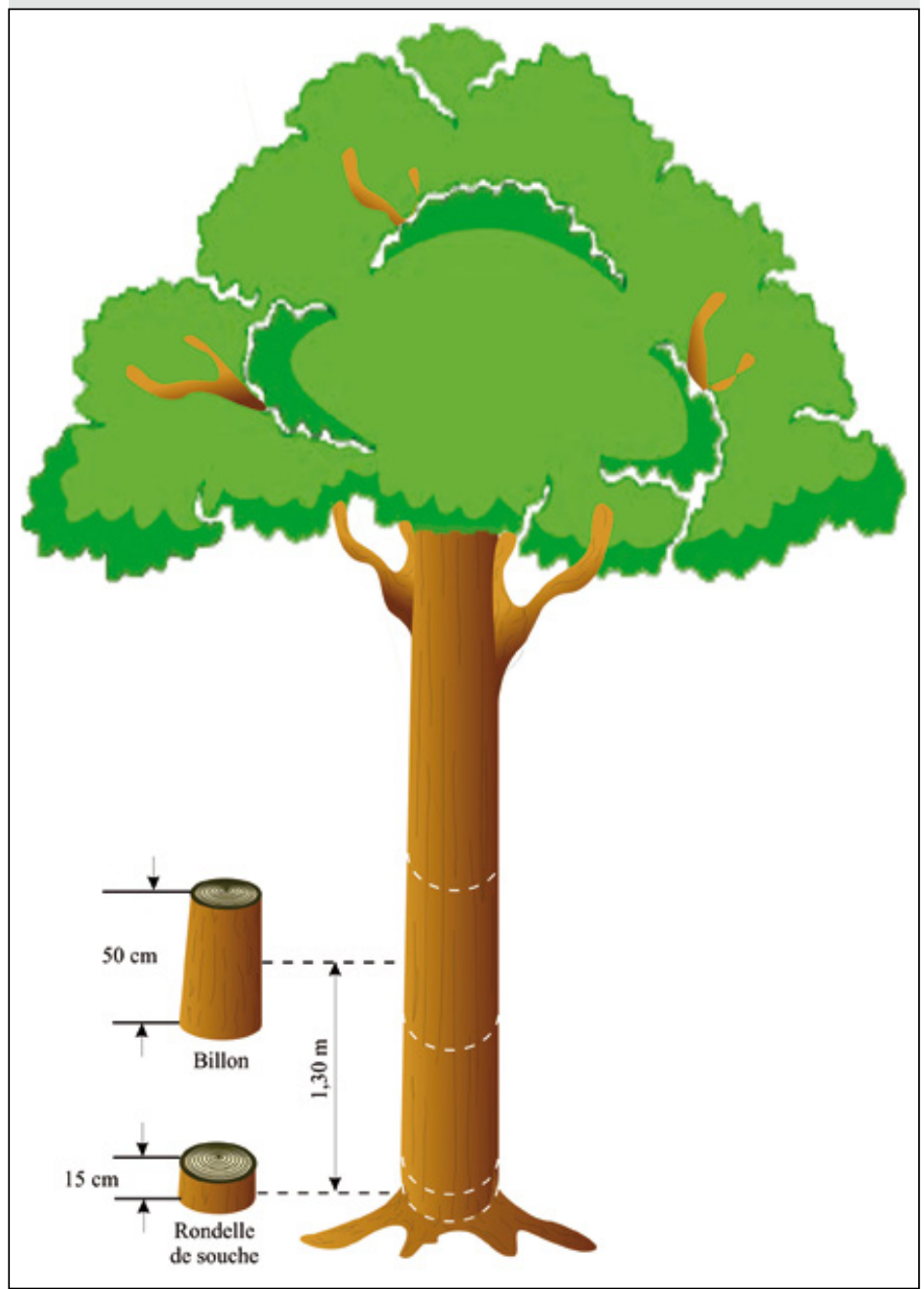

Figure 2.

Débit d'un billon et d'une rondelle de souche

à partir d'un pistachier de l'Atlas.

Tableau I.

Données dendrométriques des arbres mesurés sur terrain.

\section{Localisation} des régions

Tiaret (forêt de Tagdempt)

\section{Situation géographique} (cordonnées GPS)

Latitude : 35.357207

Longitude : 1290631

Altitude : $1008 \mathrm{~m}$

\section{Exposition}

Versant sud-est

Sidi Bel Abbès Latitude : 35.137125 Plat (région de M'cid) Longitude :-0,247719 Altitude : $420 \mathrm{~m}$

\begin{tabular}{|c|c|c|c|c|c|c|c|c|c|}
\hline \multirow{2}{*}{$\begin{array}{l}\text { Type } \\
\text { de sol }\end{array}$} & \multirow{2}{*}{$\begin{array}{l}\text { Numéro } \\
\text { d'arbre }\end{array}$} & \multirow{2}{*}{$\begin{array}{l}\text { Âge } \\
\text { (ans) }\end{array}$} & \multirow{2}{*}{$\begin{array}{l}\text { Altitude } \\
\text { (m) }\end{array}$} & \multicolumn{3}{|c|}{ Circonférence (cm) } & \multicolumn{2}{|c|}{ Hauteur (m) } & \multirow{2}{*}{$\begin{array}{l}\text { Diamètre du } \\
\text { houppier }(\mathrm{m})\end{array}$} \\
\hline & & & & à la base & à 1,30 & $1^{\text {re }}$ fourche & $1^{\text {re }}$ fourche & Total & \\
\hline \multicolumn{10}{|l|}{ Brun } \\
\hline & 1 & 50 & 750 & 66 à 0,2 & 51 & 55 & 1,84 & 4,34 & 3,70 \\
\hline & 2 & 37 & 760 & 53 à 0,1 & 44 & 40 & 1,70 & 5,65 & 3,50 \\
\hline & 3 & 47 & 770 & 90 à 0,3 & 70 & 80 & 1,80 & 6,14 & 5,80 \\
\hline \multicolumn{10}{|l|}{ Brun } \\
\hline & 1 & 24 & 420 & 68 à 0,1 & 57 & 67 & 1,81 & 3,23 & 4,70 \\
\hline & 2 & 51 & 420 & 82 à 0,1 & 58 & 68 & 1,90 & 4,02 & 4,20 \\
\hline & 3 & 48 & 420 & 77 à 0,2 & 59 & 54 & 1,85 & 4,85 & 4,70 \\
\hline
\end{tabular}




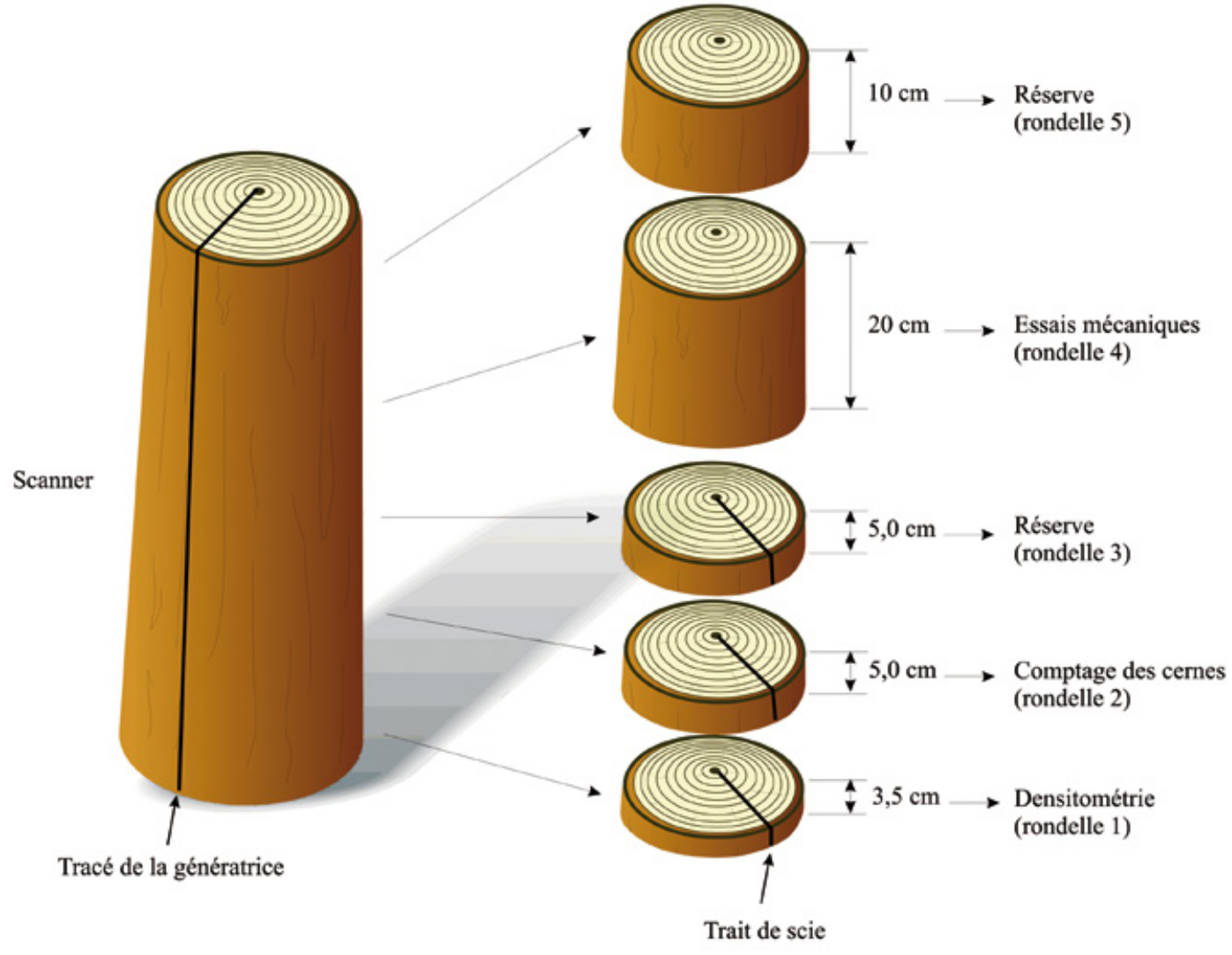

Figure 3.

Découpe d'un billon en cinq rondelles.

\section{Découpe des billons en rondelles}

Après l'établissement du plan de découpe et de repérage, un rayon passant par la moelle (le plus grand rayon sur sa face supérieure), puis une génératrice ont été tracés tout au long de chaque billon. Chaque billon a été découpé en cinq rondelles en allant du bas vers le haut (Gelhaye et Mothe, 2006). Au total, nous avons collecté 30 rondelles par cette opération, plus les six rondelles de souche (figure 3 ). Pour chaque lot de cinq rondelles :

- une rondelle $n^{\circ} 1$ de $3,5 \mathrm{~cm}$ a été découpée en éprouvettes de $2 \mathrm{~mm}$ d'épaisseur destinées aux mesures microdensitométriques;

- une rondelle $\mathrm{n}^{\circ} 2$ de $5 \mathrm{~cm}$ a été destinée au comptage du nombre de cernes et à la mesure de leur largeur; sur chaque rondelle, une fente radiale a été sciée suivant la génératrice en passant par la moelle ;

- une rondelle $\mathrm{n}^{\circ} 3$ de $5 \mathrm{~cm}$, sciée de façon similaire à la précédente, a été gardée à titre conservatoire ;

- une rondelle $\mathrm{n}^{\circ} 4$ de $20 \mathrm{~cm}$ a été destinée aux essais mécaniques et aux mesures colorimétriques ;

- et enfin, une rondelle $\mathrm{n}^{\circ} 5$ de $10 \mathrm{~cm}$ a été isolée à titre conservatoire.

Chaque rondelle a ensuite été immatriculée sur sa face supérieure par la lettre $\mathrm{S}$ ou $\mathrm{T}$ indiquant la provenance, suivie du numéro d'arbre (1 à 3), du numéro de rondelle (1 à 5 du bas vers le haut), et enfin du numéro de rayon (1 à 4). Par exemple, $S_{3.4 .1}$ nous indique qu'il s'agit de l'arbre provenant de Sidi Bel Abbès, $n^{\circ} 3$, rondelle 4, rayon 1.
La rondelle de souche de $15 \mathrm{~cm}$, sur la face inférieure de laquelle deux rayons non opposés ont été tracés, a été destinée au comptage du nombre de cernes pour estimer l'âge de l'arbre (Gelhaye et Mothe, 2006).

\section{Mode de prélèvement des éprouvettes}

Le prélèvement des éprouvettes a été fait au LERFoB en respectant au mieux l'orientation principale du fil.

Les éprouvettes coupées au niveau du duramen de l'aubier et de la zone de transition sont celles ayant préalablement servi pour des essais mécaniques (figure 4). Ainsi, sur la face inférieure des rondelles de bois de $20 \mathrm{~cm}, 12$ éprouvettes de $2 \mathrm{~mm}$ d'épaisseur dans le sens radial et $12 \mathrm{~mm}$ de largeur dans le sens tangentiel ont été prélevées sur deux des plus grands rayons tracés sur chaque rondelle, en évitant les défauts apparents (photo 3). Le nombre total des éprouvettes était de 72 (tableau II) ; les éprouvettes mesurées du duramen, de l'aubier et de la zone de transition présentaient une densité moyenne respective de 1,14, 0,85 et 1,01.

La distance à la moelle a été relevée pour chaque éprouvette. Le rapport entre cette distance et la longueur radiale totale de l'éprouvette a été utilisé pour identifier approximativement le cerne correspondant à chaque éprouvette dans les comptages de cernes réalisés sur la rondelle $n^{\circ} 4$. L'âge compté depuis la moelle et la largeur du cerne considéré a été relevé. 
Figure 4.

Mode opératoire de la préparation des éprouvettes suivant les quatre rayons tracés à partir de la rondelle $n^{\circ} 4$.

mesurage et l'affichage des données. La fenêtre utilisée pour mesurer la couleur avait une ouverture de $8 \mathrm{~mm}$ de diamètre (photo 4). Le spectrocolorimètre analysait, longueur d'onde par longueur d'onde, l'énergie lumineuse réfléchie ou transmise afin de déterminer les courbes spectrales d'un objet. Une fois calibré, l'appareil a été déposé sur la surface longitudinale-tangentielle des éprouvettes le long desquelles 10 positions équidistantes ont été repérées. Deux mesures répétées par position ont été effectuées, soit 1440 mesures (72 éprouvettes $x 10$ positions $\times 2$ mesures). Les composantes colorimétriques $L^{*}, a^{\star}$ et $b^{\star}$, qui correspondent respectivement à la clarté, à l'axe vert-rouge, et à l'axe bleu-jaune, ont été mesurées dans le système CIELab sur les éprouvettes. L'écart-type entre les mesures (moyennes des deux répétitions) effectuées sur les 10 positions de chaque éprouvette nous a servi à caractériser l'hétérogénéité locale de la couleur. Ces mesures ont été corrélées avec la densité du bois afin de montrer les variations de la couleur en fonction de la provenance des arbres, des arbres eux-mêmes et du type de bois (aubier ou duramen). Le bois mixte, représenté par la zone de transition, a également été pris en considération dans les mesures.

\section{Principe du système CIELab 1976}

Après sciage, les éprouvettes ont été séchées à l'air libre pendant environ quatre semaines dans des conditions non régulées au LERFoB. La valeur finale d'humidité obtenue sur ces éprouvettes était de $12 \%$.

\section{Appareils de mesure}

Deux principaux types d'appareils sont utilisés pour mesurer la couleur : les colorimètres et les spectrocolorimètres. Ces appareils sont équipés d'un référentiel de couleur normalisé au niveau international. Le colorimètre est principalement utilisé en contrôle qualité afin d'obtenir des valeurs numériques et de vérifier la comparabilité avec des tolérances définies. La mesure de la couleur du bois et de ses variations a été réalisée à l'aide d'un spectrocolorimètre relié à une unité de calcul avec un logiciel afin de permettre le
Dans l'espace CIELab 1976, défini par la Commission internationale de l'éclairage (CIE), le plan chromatique est défini par ses axes vert-rouge et bleu-jaune (figure 5). L'axe perpendiculaire à ce plan et passant par l'intersection des deux axes définit la clarté. Selon Chrisment (1997), ce système est destiné à l'étude des couleurs de surface et d'objet à l'aide des coordonnées chromatiques qui permettent de quantifier la couleur en chaque point par trois composantes $a^{*}, b^{*}$ et $L^{*}$ où :

- $a^{\star}$ est la coordonnée chromatique correspondant à l'axe vert-rouge ;

- $b^{\star}$ est la coordonnée chromatique correspondant à l'axe bleu-jaune ;

- L* est la clarté (luminance) variant de 0 (désignant un noir parfait) à 100 (désignant un blanc immaculé).

Tableau II.

Nombre de chaque type d'éprouvettes par rayon et par région.

\begin{tabular}{|c|c|c|c|c|c|c|c|c|c|c|}
\hline \multirow[t]{2}{*}{ Type d'éprouvettes } & & & Tiaret & & & & \multicolumn{3}{|c|}{ Sidi Bel Abbès } & \multirow[b]{2}{*}{ Total } \\
\hline & R1 & R2 & R3 & R4 & Total & R1 & R2 & R3 & R4 & \\
\hline Duramen & 02 & 02 & - & 03 & 07 & 05 & 02 & - & 06 & 13 \\
\hline Aubier & 09 & 04 & 04 & 04 & 21 & 07 & 02 & - & 08 & 17 \\
\hline Mixte & 03 & 01 & 01 & 03 & 08 & 02 & 01 & - & 03 & 06 \\
\hline
\end{tabular}



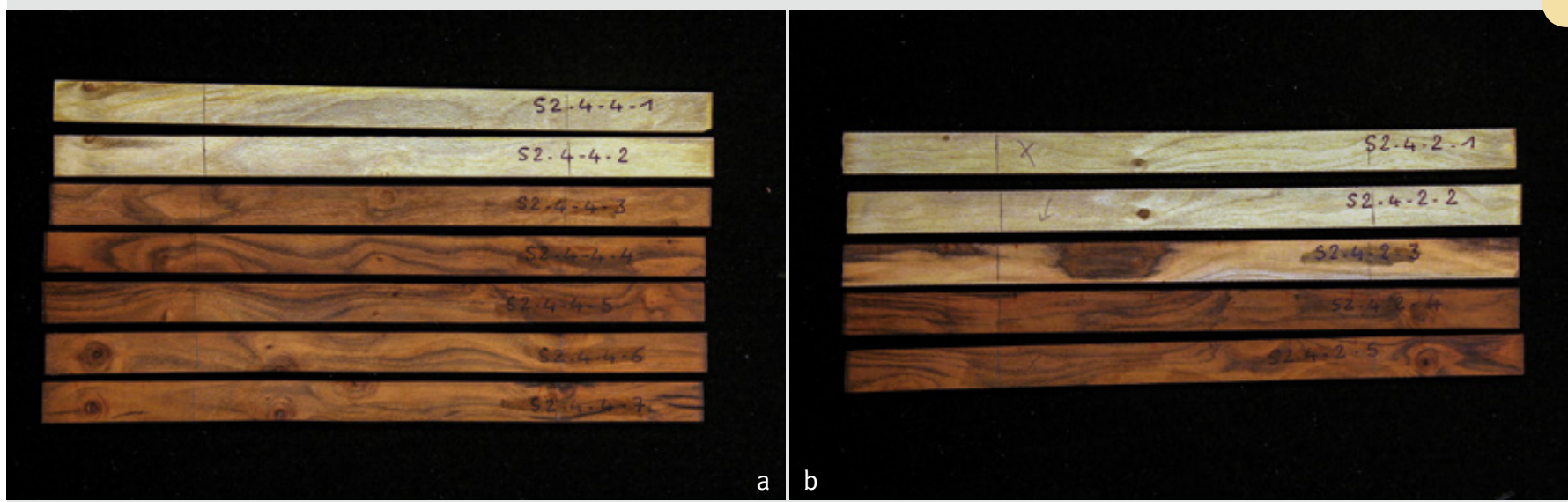

Photo 3.

Les 12 éprouvettes prélevées dans l'aubier et le duramen sur les deux rayons d'une rondelle de pistachier de l'Atlas.

Sept éprouvettes prélevées du rayon S2.4.4.1 (a) ; cinq éprouvettes prélevées du rayon S2.4.2.1 (b).

Photo M. Boudouaya.

Les résultats de la couleur d'un objet peuvent être également exprimés à l'aide de deux autres composantes calculables à partir de ces composantes de base $a^{*}$ et $b^{\star}$ : - la composante chromatique $C^{*}$ (chroma), ou « saturation de la teinte » de l'objet coloré, correspond à l'intensité de la teinte par rapport à la teinte pure de référence située sur le cercle chromatique ; elle représente la distance à l'axe blanc-noir : $C^{*}=\sqrt{ }\left(a^{2}+b^{2}\right)$;

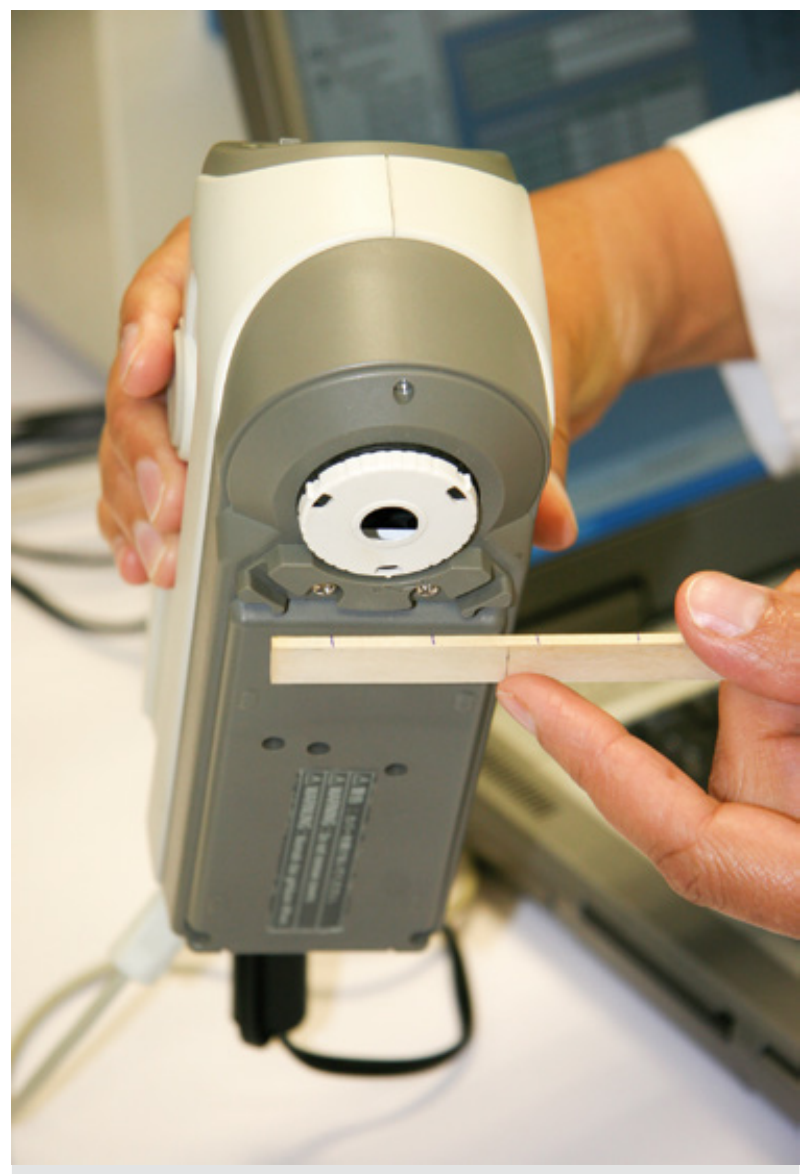

Photo 4.

Mesure de la couleur sur les éprouvettes. Photo M. Boudouaya.
- la composante chromatique $\mathrm{h}^{\star}$ (hue) correspond à l'« angle de teinte » qui est une estimation de la distance angulaire par rapport à l'axe rouge $: h^{\star}=\arctan (b / a)$.

\section{Mesures et analyses statistiques}

Les résultats numériques obtenus sur les caractéristiques colorimétriques ont été traités statistiquement par une analyse de la variance afin de ressortir les variabilités inter-arbres et inter-provenances d'une part ; d'autre part, ils ont été représentés graphiquement avec la densité en utilisant les logiciels d'analyse statistique Statistica version 06 et $R$ (R Core Team, 2014).

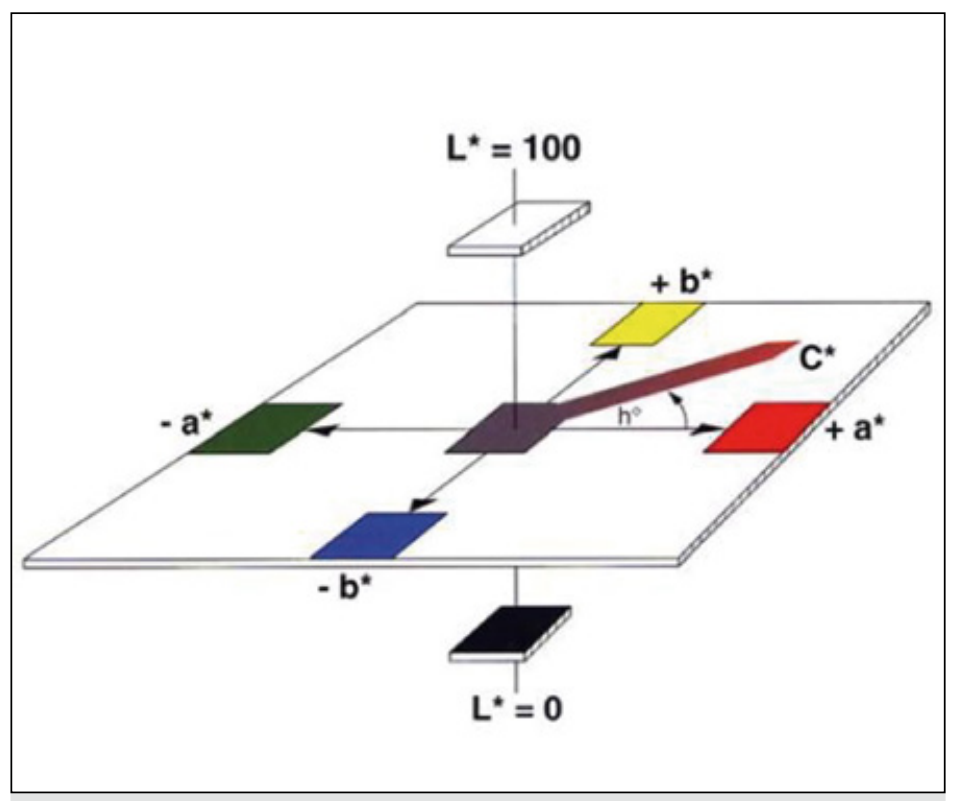

Figure 5.

Espace CIELab (1976) (Chrisment, 1997). 


\section{Résultats}

\section{Différences entre aubier, duramen et type mixte}

Les variations avec la densité de la clarté $L^{*}$, des composantes $a^{\star}$ et $b^{\star}$, de l'écart-type de la clarté, de la saturation $C^{\star}$ et de l'angle de teinte $h^{\star}$ pour l'aubier, le duramen et le type mixte pour l'ensemble des éprouvettes des deux provenances considérées, sont illustrées dans la figure 6 . Les résultats détaillés sont présentés dans le tableau III.

Ces résultats montrent clairement une différence significative de couleur entre l'aubier et le duramen pour les deux provenances.

La composante clarté $L^{*}$ est significativement plus élevée dans l'aubier (entre 73,88, valeur minimale, et 82,03 , valeur maximale) que dans le duramen (entre 51,05 et 62,58$)$; au contraire, la valeur de la composante $a^{*}$ est significativement plus élevée dans le duramen (entre 10,53 et 11,67 ) que dans l'aubier (entre 6,11 et 7,44 ) (tableau III).

Pour la composante $b^{\star}$, la différence entre les trois types du bois du pistachier est globalement non significative $(p=0,0192$ pour la première provenance et $p=0,08106$ pour la seconde).

La saturation $C^{\star}$ ne permet pas non plus de discriminer les trois types, au contraire de l'angle de teinte $h^{\star}$ qui est systématiquement plus élevé dans l'aubier que dans le duramen.

Les éprouvettes mixtes, qui comprennent à la fois de l'aubier et du duramen, présentent des caractéristiques intermédiaires comme le montrent le tableau III et la figure 6 , et une hétérogénéité plus forte dans l'échantillon, traduite par l'écart-type de clarté (figure 6D).

\section{Relation entre couleur et densité du bois}

Le duramen présente une densité plus forte que l'aubier. La figure 6 montre qu'il existe une forte corrélation entre la densité d'une part et la clarté $L^{*}\left(r=-0,893^{\star * \star}\right)$, la composante $a^{\star}\left(r=0,759^{\star \star \star}\right)$ et l'angle de teinte $h^{\star}$ $\left(r=-0,789^{\star \star \star}\right)$ d'autre part. Par contre, il n'y a pas ou peu de corrélation de la densité avec la composante $b^{\star}$ $(r=-0,018)$ ou la saturation $C^{*}\left(r=0,265^{\star}\right)$.

La plupart de ces tendances ne se confirment pas lorsque l'on observe séparément l'aubier et le duramen. Dans l'aubier, la densité n'est significativement corrélée qu'à $L^{\star}\left(r=-0,465^{\star}\right)$ et $h^{\star}\left(r=0,452^{\star}\right)$. Dans le duramen, c'est la composante $b^{\star}$ qui est la mieux corrélée à la densité $\left(r=-0,779^{\star \star \star}\right)$, suivie de $C^{\star}\left(r=-0,737^{\star \star \star}\right)$ et $h^{\star}$ $\left(r=-0,574^{*}\right)$.

\section{Relation entre couleur et âge et largeur de cerne}

La figure 7 présente les relations entre composantes chromatiques d'une part, âge et largeur du cerne correspondant approximativement à l'éprouvette d'autre part.

En moyenne, on observe une légère augmentation de la clarté avec l'âge du cerne $\left(r=0,342^{\star *}\right)$. L'effet de l'âge est cependant différent dans l'aubier, où la clarté n'augmente pas significativement avec l'âge $(r=0,127)$ et dans le duramen où elle décroît $\left(r=-0,559^{\star}\right)$. On observe un comportement analogue pour la largeur de cerne qui n'est pas corrélée en moyenne avec la clarté $(r=0,070)$, alors que la relation est négative dans l'aubier $\left(r=-0,454^{*}\right)$ et plutôt positive dans le duramen $(r=0,386)$.

La composante $a^{*}$ tend à décroître avec la largeur de cerne $(r=-0,224)$ et surtout avec l'âge $\left(r=-0,401^{\star \star \star}\right)$. La corrélation de $a^{\star}$ avec la largeur de cerne est significative dans l'aubier $\left(r=-0,362^{*}\right)$ et dans le duramen $\left(r=-0,542^{\star}\right)$, au contraire de celle de $a^{\star}$ avec l'âge qui ne se confirme ni dans l'aubier $(r=0,006)$ ni dans le duramen $(r=0,031)$.

En ce qui concerne la composante $b^{\star}$, aucune relation significative avec l'âge ou la largeur de cerne n'a été mise en évidence.

\section{Différences entre arbres et entre provenances}

La figure 6 et le tableau III montrent également les différences entre les deux provenances. On observe que le bois provenant de Tiaret est plus clair que celui de Sidi Bel Abbès, notamment dans l'aubier $\left(p<10^{-6}\right)$. Les teintes sont presque identiques dans les deux provenances bien que des différences faiblement significatives soient obtenues dans l'aubier pour les composantes $a^{\star}$ et $b^{\star}$.

Le tableau III montre enfin les différences entre les arbres de la même provenance. On observe une différence significative au seuil de $5 \%$ pour la clarté entre les arbres de Sidi Bel Abbès pour l'aubier et le duramen ( $p=0,012$ et $p=0,018$ respectivement). La différence est aussi significative entre les arbres de Tiaret pour l'aubier $(p=0,00012)$ mais non significative pour le duramen.

\section{Discussion et conclusion}

Cette étude colorimétrique effectuée sur le bois du pistachier de l'Atlas a montré une différence importante entre l'aubier et le duramen pour les composantes $L^{\star}$ et $a^{\star}$ ainsi que pour l'angle de teinte $h^{\star}$ et dans une moindre mesure la saturation $C^{\star}$. Ainsi, comparé à l'aubier, le bois du duramen est beaucoup plus sombre et sa couleur tend davantage vers le rouge.

La clarté moyenne du duramen de Pistacia atlantica Desf. était de 54,9 (dans le plan LT), ce qui le classe parmi les bois très sombres, comparé par exemple au duramen de chêne (72,2 pour Quercus petraea d'après Klumpers et al., 1993). Cette valeur est voisine de celles obtenues par Garcia et al. (2014) sur Eucalyptus paniculata (51,2 dans le plan LT, 54,5 dans le plan LR), par Amusant et al. (2004) sur du duramen de Dicorynia guianensis Amsh (56,2 dans les plans LT et LR), ou encore par Sotelo Montes et al. (2013) sur Guiera senegalensis (54,9 dans le plan RT). En revanche, d'après les mêmes auteurs, la teinte de Pistacia atlantica ( $a^{\star}=11,4$ et $\left.b^{\star}=22,7\right)$ le rapproche beaucoup plus de Quercus petraea $\left(a^{*}=10\right.$ et $\left.b^{*}=22,8\right)$ que d'Eucalyptus paniculata $\left(a^{\star}=16,5\right.$ et $b^{\star}=18,0$ dans le plan LT) ou de Dicorynia guianensis ( $a^{*}=15,3$ et $\left.b^{*}=23,5\right)$, qui sont des bois plus rouges, ou de Guiera senegalensis $\left(a^{*}=5,7\right.$ et $\left.b^{\star}=15,8\right)$ dont la couleur tire davantage sur le vert. Ces comparaisons 
A

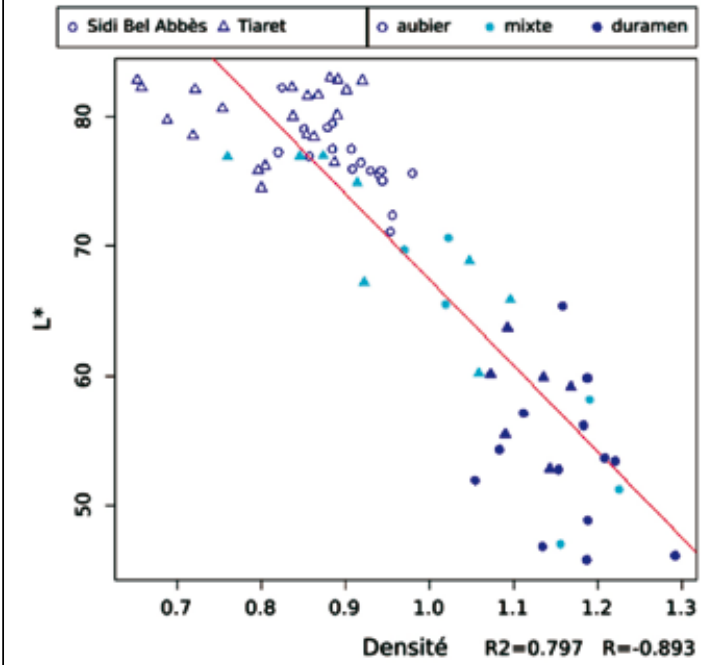

D

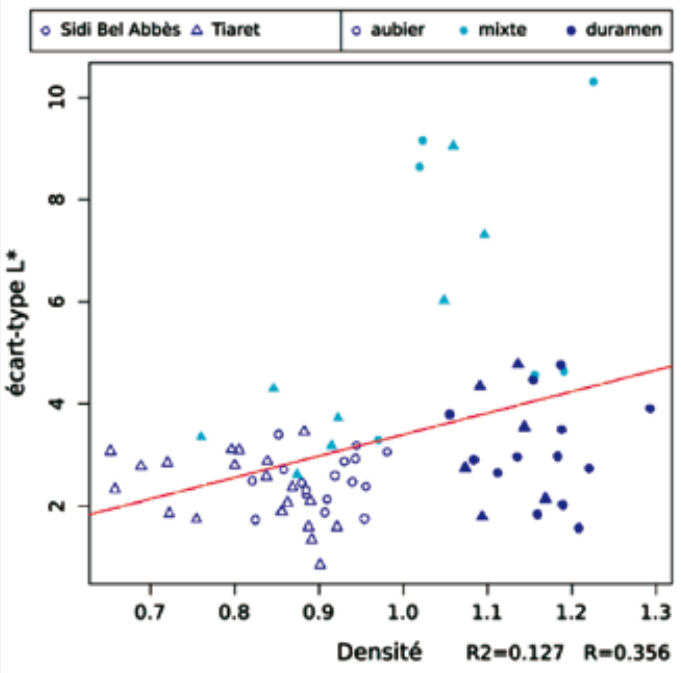

B

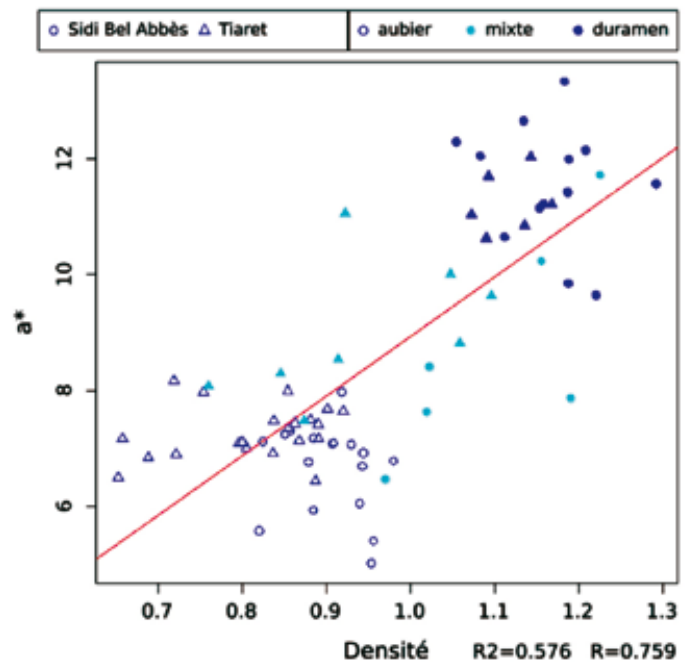

E

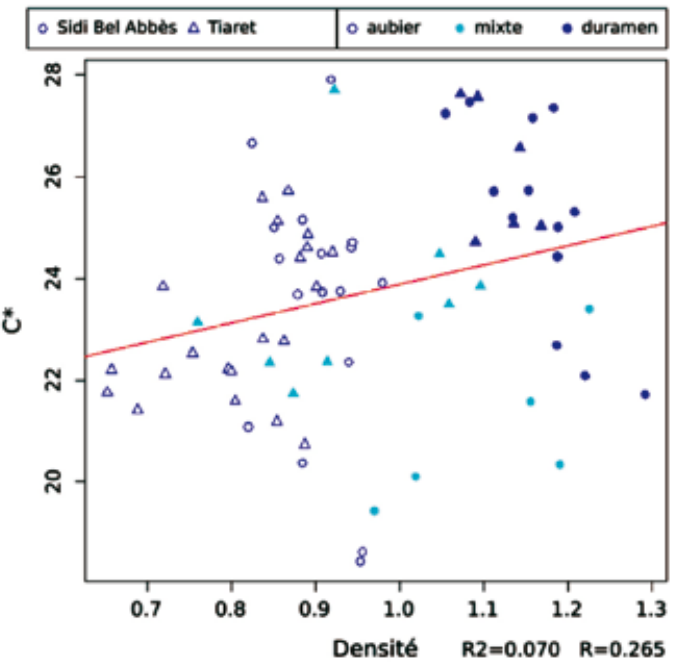

C

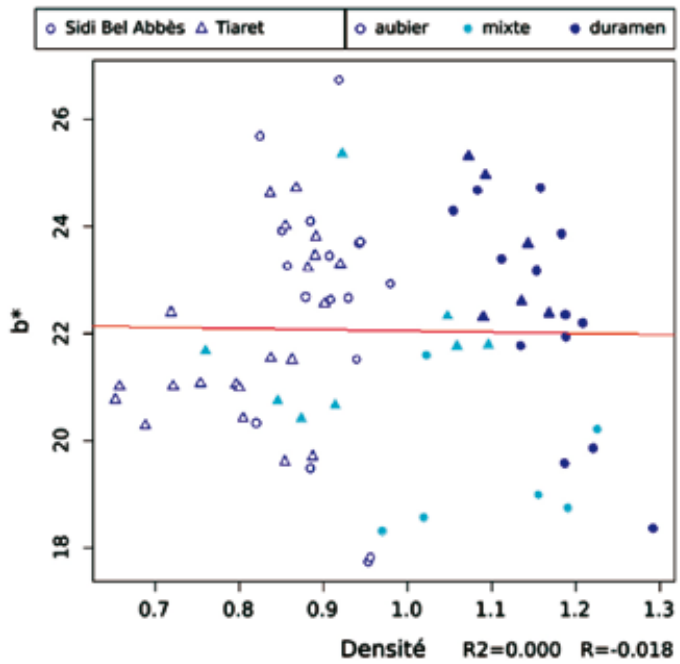

F

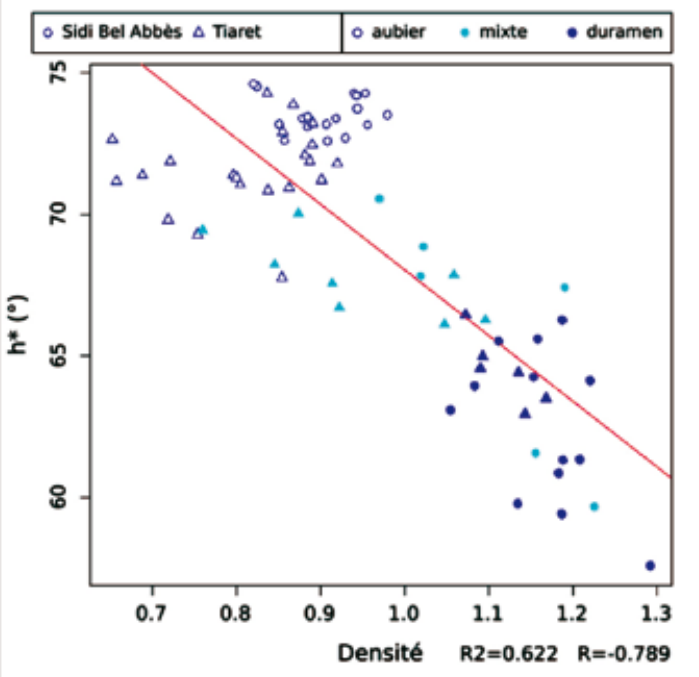

Figure 6.

Variations avec la densité de la clarté $L^{\star}$, des composantes $a^{\star}$ et $b^{\star}$, de l'écart-type de la clarté, de la saturation $C^{\star}$ et de l'angle de teinte $h^{\star}$ pour l'aubier, le duramen et le type mixte. 
Tableau III.

Valeurs moyennes des mesures de $L^{*}, a^{*}$ et $b^{*}$ effectuées sur les 72 éprouvettes issues des trois arbres des deux provenances et résultats de l'analyse des variances au seuil de $5 \%$.

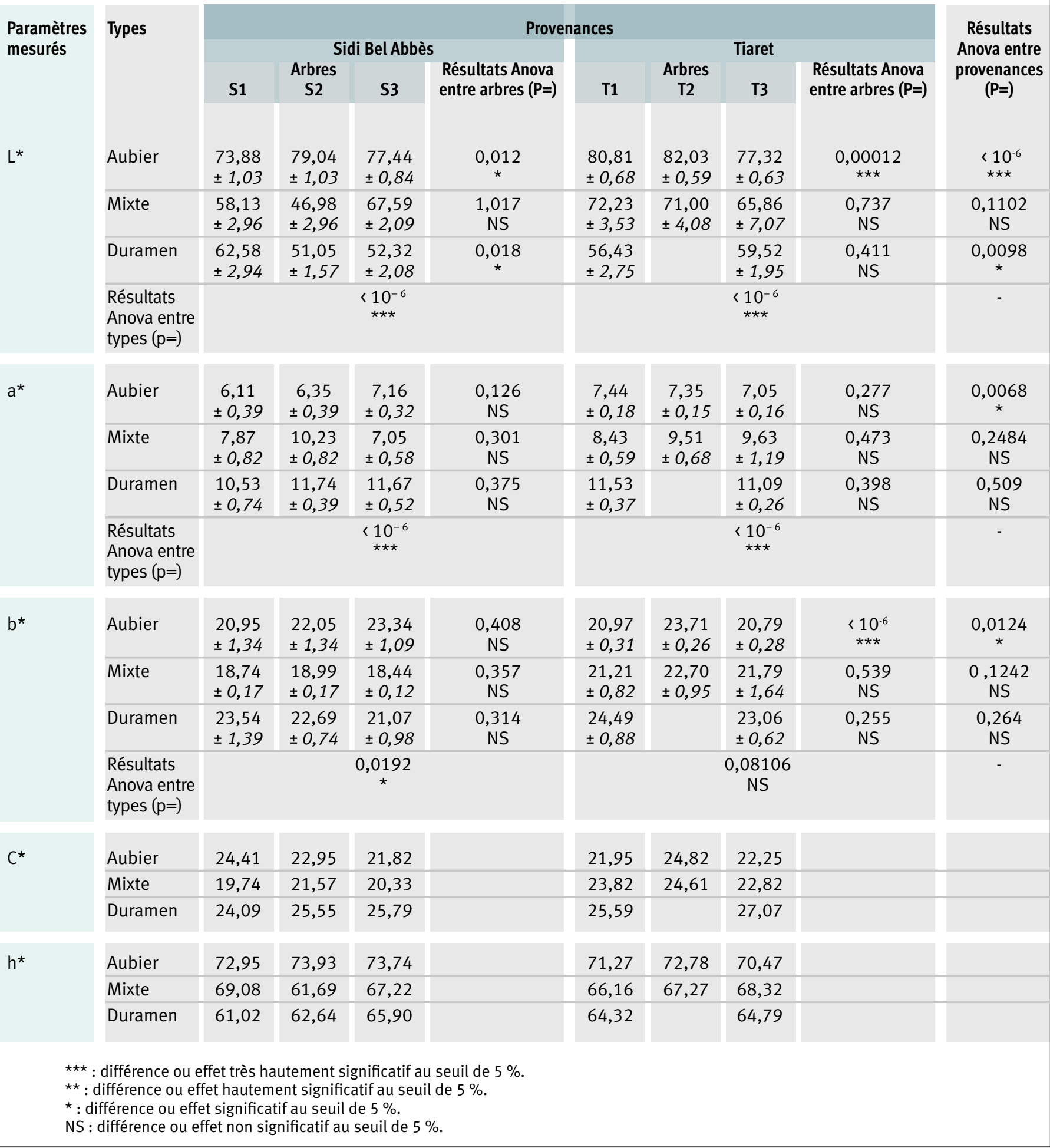




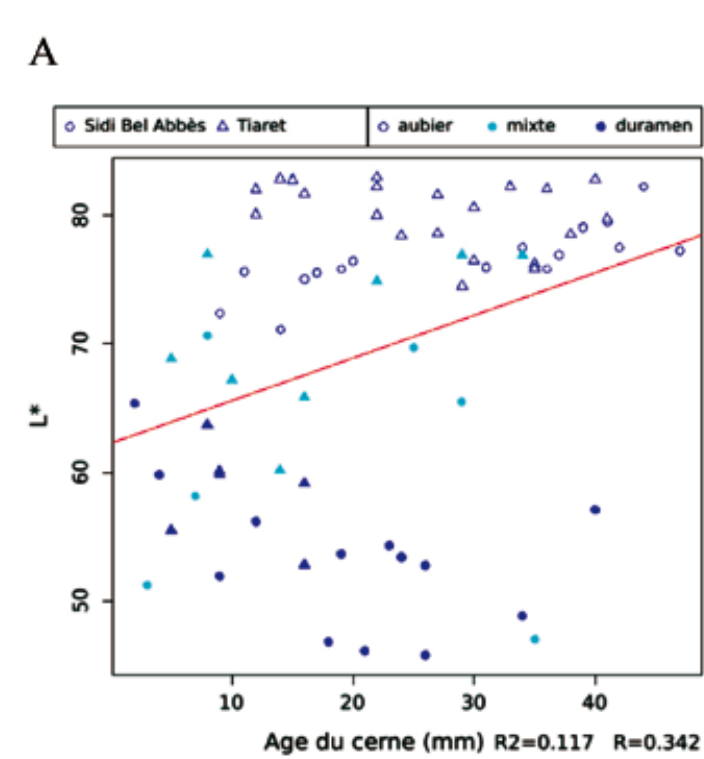

D
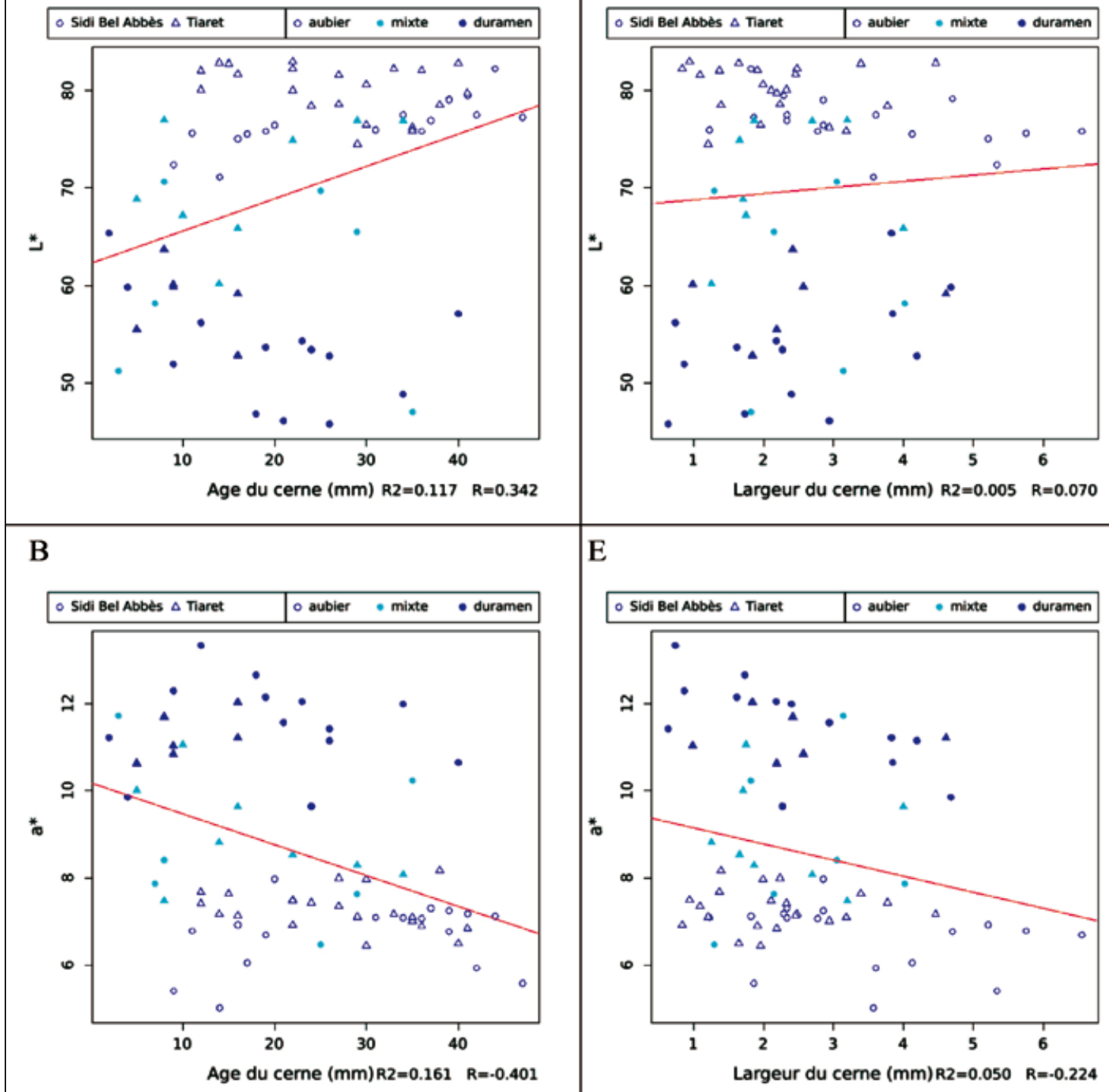

E

$\mathrm{C}$
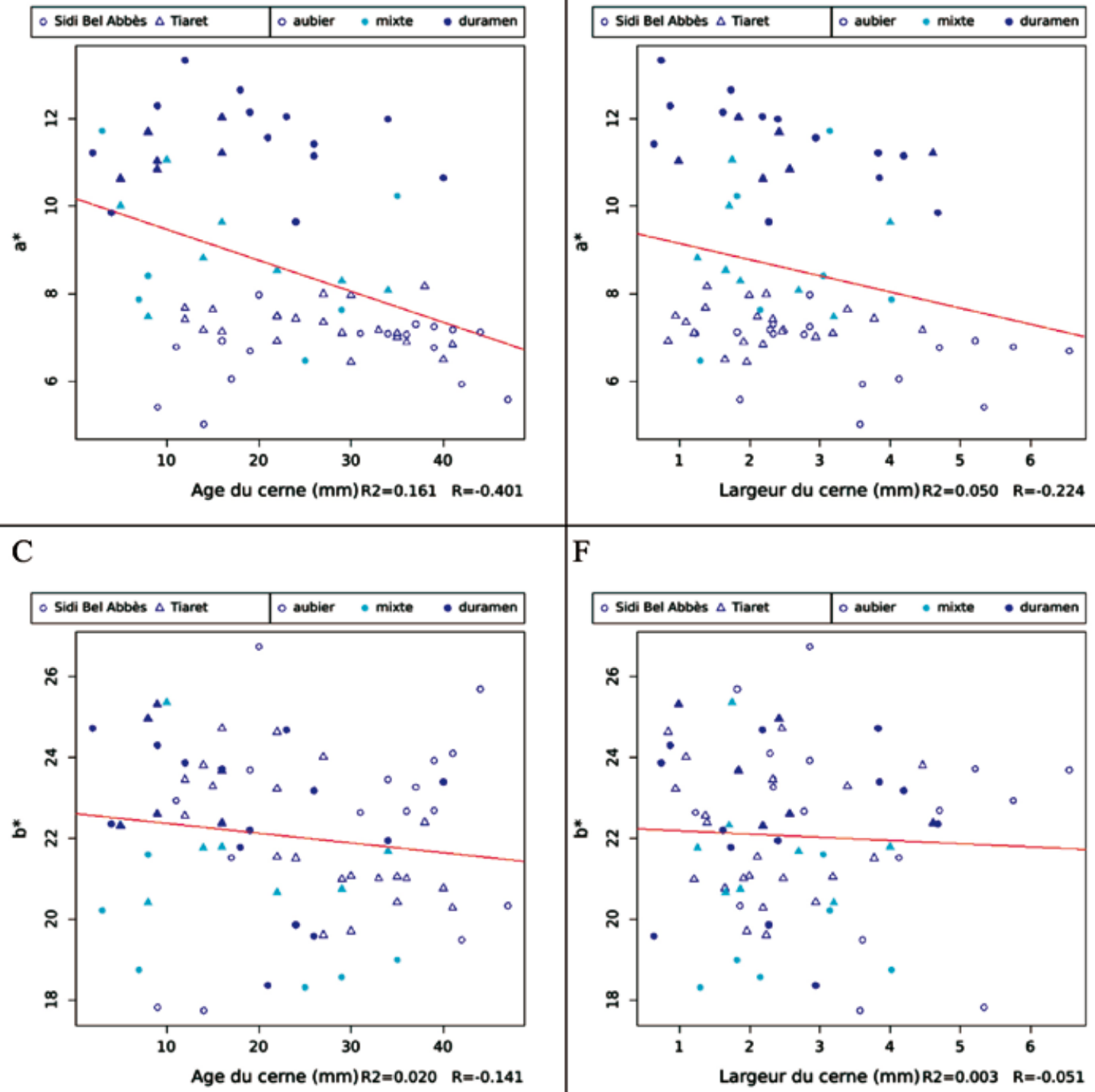

F

Figure 7.

Variations avec l'âge compté depuis la moelle et la largeur du cerne contenant l'éprouvette avec la clarté L* et les composantes $a^{\star}$ et $b^{\star}$ pour l'aubier, le duramen et le type mixte. 
sont toutefois à considérer avec précaution puisque la couleur dépend non seulement de l'orientation des éprouvettes mais aussi de la durée et des conditions de conservation et de séchage (Bakour, 2003 ; Amusant et al., 2004).

L'assombrissement du bois de Pistacia atlantica au cours de la duraminisation s'explique probablement par le dépôt d'extractibles comme en témoigne l'accroissement spectaculaire de la densité qui passe en moyenne de 0,85 dans l'aubier à 1,15 dans le duramen. Ces différences expliquent les relations observées sur l'ensemble des éprouvettes entre la densité du bois et les composantes de la couleur. Les tendances observées dans l'aubier et dans le duramen entre la densité et les composantes de la couleur ne se confirment pas dans la littérature. Garcia et al. (2014) signalent ainsi une corrélation négative entre densité et $L^{*}$ sur des éprouvettes provenant de quatre espèces feuillues. Sur de jeunes spécimens de Calycophyllum spruceanum, Sotelo Montes et al. (2008) observent une relation négative entre densité et $L^{*}$ et positive entre densité et $a^{*}$ ou $b^{\star}$.

L'effet de l'âge du cerne compté depuis la moelle que nous observons dans le duramen se traduit par une tendance du bois à s'assombrir et à s'éloigner du rouge au fur et à mesure qu'il s'écarte de la moelle. La réduction de la clarté entre la moelle et la limite aubier - duramen avait déjà été observée sur différentes essences (Kokutse et al., 2006 ; Bakour, 2003 ; Amusant et al., 2004). Cet assombrissement traduit probablement un accroissement de la concentration en extractibles dans le duramen le plus externe. En admettant qu'une partie de ces extractibles contribue à améliorer la résistance du bois aux pathogènes, cette hypothèse est confortée par Amusant et al. (2004) et Kokutse et al. (2006), qui observent un accroissement de durabilité du bois entre la moelle et le duramen externe. La relation négative entre L* et le taux d'extractible n'est cependant pas vérifiée systématiquement : dans le cas du chêne, Klumpers et al. (1993) observaient bien un accroissement du taux d'extractible et une réduction de $L^{*}$ entre aubier et duramen, mais aussi une augmentation des deux variables entre la moelle et le duramen externe.

Sur le chêne également, Bakour (2003) a analysé l'effet de l'âge et de la largeur de cernes sur la couleur du duramen de Quercus robur et Quercus petrea, Il observe notamment une relation entre $b^{*}$ et la largeur de cerne (négative) et l'âge du cerne (positive) sur Quercus robur et une relation entre $\mathrm{h}^{\star}$ et la largeur de cerne (positive) et l'âge du cerne (négative) sur Quercus petraea. Sur Tetraclinis articulata, Dakak (2002) a observé des résultats plus proches de ceux que nous avons obtenus pour le duramen de Pistacia atlantica. Dans les deux cas, le bois devient plus clair et perd sa couleur rougeâtre quand l'accroissement annuel est important. Cependant, ces comportements contrastés entre espèces traduisent probablement des évolutions anatomiques ou chimiques différentes suivant les espèces avec l'âge ou la vitesse de croissance, et dont les conséquences sur la couleur sont également variables suivant les espèces.

Nos résultats montrent également une relative homogénéité de la couleur entre les arbres issus d'une même provenance, mais aussi une différence de couleur entre les deux provenances étudiées, notamment pour la clarté. Les deux provenances diffèrent du point de vue du sol, du climat et de l'altitude. La différence de clarté, relativement forte dans l'aubier, tend à s'atténuer avec la duraminisation. Sur quatre des cinq essences maliennes qu'ils ont analysées, Sotelo Montes et al. (2013) observaient pourtant une variabilité plus importante entre provenances qu'ils ont pu expliquer par des facteurs environnementaux comme le type de sol et le taux de précipitations. Les écarts entre provenances pourraient aussi s'expliquer par des différences génétiques entre les deux populations, bien que Sotelo Montes et al. (2008) aient montré, dans le cas d'une essence péruvienne, que l'héritabilité de la couleur était faible.

Nous espérons que la contribution du présent travail à l'évaluation de la couleur du bois du pistachier de l'Atlas pourra servir de référence aux futurs travaux scientifiques tendant à valoriser le bois de cette essence en se basant sur l'aspect esthétique (dessin et couleur) et son appréciation par les professionnels et tous les acteurs de la filière bois dans les pays où cet arbre prospère comme c'est le cas en Algérie.

D’autres études technologiques (usinabilité, façonnage, teinture des pièces de bois destinées aux lambris et panneaux pour la décoration) ou portant sur les aspects physiques (stabilité dimensionnelle, dureté en particulier pour le parquet) devront être menées pour une meilleure appréciation du bois du pistachier de l'Atlas dans les zones arides ou semi-arides.

\section{Références bibliographiques}

Abdelli N., 2002. Contribution à l'étude de création d'un verger dans la zone steppique de Rechaïga. Mémoire de fin d'études pour l'obtention du diplôme d'ingénieur d'État en biologie, Département des sciences agronomiques et biologiques, Université Ibn Khaldoun de Tiaret, Algérie, 125 p.

Agence nationale des ressources hydrauliques, 2013. Données climatiques sur la wilaya de Sidi Bel Abbès et de Tiaret.

Amusant N., Beauchêne J., Fournier M., Janin G., Thévenon M.-F., 2004. Decay resistance in Dicorynia guianensis Amsh.: analysis of inter-tree and intra-tree variability and relations with wood colour. Annals of Forest Sciences, 61: 373-380.

Bakour R., 2003. Influence de l'espèce et de la provenance des deux principaux chênes français (Quercus robur L. ; Quercus petraea Liebl.) sur la structure anatomique et les propriétés physiques du bois de merrain. Thèse, École nationale du génie rural, des eaux et forêts, Nancy, France, 247 p.

Chrisment A., 1997. Couleur et colorimétrie. Éditeur : 3C Conseil, $30 \mathrm{p}$. 
Dakak J., 2002. La qualité du bois de Thuya de Maghreb (Tetraclinis articulata) et ses conditions de développement sur ses principaux sites phytoécologiques de son bloc méridional au Maroc. Thèse de docteur en sciences forestières et du bois, Engref, Centre de Nancy, France, 149 p.

Direction des services agricoles de Sidi Bel Abbès, 2008. Données climatiques de la station météorologique de la wilaya de Sidi Bel Abbès (Algérie).

Dumon R., 1980. La forêt, source d'énergie et d'activités nouvelles. Paris, Masson, coll. Objectifs scientifiques de demain, $4^{\mathrm{e}}$ édition, $145 \mathrm{p}$.

Garcia R. A., Silveira de Oliveira N., Do Nascimento A. M., Dias de Souza N., 2014. Colorimetria de madeiras dos gêneros eucalyptus e corymbia e sua correlação com a densidade. Universidade Federal de Lavras, Brasil, Cerne, vol. 20, núm. 4, p. 509-517.

Gelhaye P., Mothe F., 2006. Densitométrie : Notices d'utilisation. Nancy, France, Inra, Laboratoire d'étude des ressources forêt-bois de l'équipe Croissance, Production et Qualité des Bois (CPQB), $21 \mathrm{p}$.

Gillet F., 2000. La phytosociologie synusiale intégrée. Guide méthodologique. Université de Neuchâtel, Institut de botanique, Suisse, Documents du Laboratoire d'écologie végétale et de phytosociologie, 1, $68 \mathrm{p}$.

Janin G., 1987. Mesure de la couleur du bois. Intérêt forestier et industriel. Annales des Sciences Forestières, 44 (4) : 455-472.

Klumpers J., Janin G., Becker M., Levy J., 1993. The influences of age, extractive content and soil water on wood color in oak: the possible genetic determination of wood color. Annales des Sciences Forestières, 50 (suppl. 1): 403-409.

Kokutse A. D., 2002. Analyse de la qualité du bois de teck (Tectona grandis L.f) en plantation au Togo : formation du bois de cœur, propriétés mécaniques et durabilité. Thèse de doctorat, Université Bordeaux I, France, 163 p.

Kokutse A. D., Stokes A., Baillères H., Kokou K., Baudasse C., 2006. Decay resistance of Togolese teak (Tectona grandis L.f) heartwood and relationship with colour. Trees, 20 (2): 219-223.

Mazet J.-F., Janin G., 1990. La qualité de l'aspect des placages de chênes : mesures de couleur et critères d'appréciation des professionnels français et italiens. Annales des Sciences Forestières, 47 (3) : 255-268.

Monjauze A., 1980. Connaissance du Bétoum. (Pistacia atlantica Desf.). Revue Forestière Française, 32 (4) : 357-363.

Quézel P., Médail F., Loisel R., Barbero M., 1999. Biodiversité et conservation des essences forestières du bassin méditerranéen. Unasylva, $197: 21-28$.

Quézel P., 2000. Réflexions sur l'évolution de la flore et de la végétation au Maghreb méditerranéen. Paris, France, Ibis Press, $117 \mathrm{p}$.
R Core Team, 2014. R: a language and environment for statistical computing. Vienne, Autriche, R Foundation for Statistical Computing. http://www.R-project.org

Sotelo Montes C., Hernandez R., Beaulieu J., Weber J. C., 2008. Genetic variation in wood color and its correlations with tree growth and wood density of Calycophyllum spruceanum at an early age in the Peruvian Amazon. New Forests, 35: 57-73.

Sotelo Montes C., Weber J. C., Garcia R. A., Silva D. A., Muniz G. I. B., 2013. Variation in wood color among natural populations of five tree and shrub species in the Sahelian and Sudanian ecozones of Mali. Journal Canadien de la Recherche Forestière, 43: 552-562.

Zanetti M., Mothe F., Merlin A., Janin G., Le Moguédec G., Goncalez J., 2003. Conséquences du vieillissement du bois de chêne sessile (Quercus petraea (Matt.) Liebl.) sur sa perception esthétique par les utilisateurs. Annals of Forest Sciences, $60: 61-68$. 